\title{
NOTES ON MALIGNANT GROWTHS IN THE INSANE.'
}

\author{
By JOHN RUDOLPH KNAPP, M. D.,
}

Assistant Physician, Manhattan State Hospital, East. Ward's Island, New York City.

Realizing the interest taken in the subject of malignant growths, and appreciating the untiring efforts of an honorable profession striving to solve the etiological problems connected therewith, the idea occurred to me-and was approved by Dr. A. E: Macdonald, superintendent of the Manhattan State Hospital, East-that a paper embodying the subject as observed in our hospital might not prove uninteresting for the consideration of the Association.

It was found from examination of the reports of the Manhattan State Hospital for eight years ending September 30, 1896 (where the total number of male and the total number of female patients under treatment were nearly equal, and the total number of male and the total number of female patients who died were also nearly equal), that one patient died from malignant growth for every 418 patients under treatment, and for every II I patients who died, one died of a malignant growth; also, that the female deaths from malignant growths exceeded those of the male in the ratio of two to one.

In the Manhattan State Hospital, East, which has been until recently the male department of the Manhattan State Hospital, it was found from examination of the records from the beginning that one patient died from malignant growth for every $626 \mathrm{pa}-$ tients under treatment, and for every 217 patients who died, one died of a malignant growth.

The twelfth census of the United States, that of 1900, shows that the death-rate from cancer and tumor is 60 to 100,000 of the population, or I to $I, 666$. However, as cancer is usually a disease of adult life, and especially so in the insane, a comparison of

${ }^{1}$ Read before the 59th Annual Meeting of the American Medico-Psychological Association, at Washington, D. C., May 12-15, 1903. 
frequency without allowing for this would perhaps be misleading. As the adult population at large is about two-thirds of the whole, an estimation on this basis would give one death from malignant growth to I,II2 of the general adult population.

It is, therefore, seen that malignant growth is more than twice as frequent among the insane as among the adult population at large, and nearly four times as frequent among the insane compared with the general population.

From the beginning, a total of 31 male patients have died from malignant growths; of this number there were 22 cases of carcinoma and nine of sarcoma, five of the latter being instances of sarcoma of the brain. As many of these cases presented special points of interest, a short detailed description may not prove uninteresting.

One Patient Died of Primary Carcinoma of the Pancreas:

An English laborer, aged 32, an advanced case of paresis of unknown duration, with a history of intemperance. One month prior to his death, he complained of acute abdominal pain; this was followed by profuse bloody diarrhoea. The neoplasm was detected in the region of the pancreas by palpation. He died of perforation of the intestine.

Two Patients Died of Epithelioma Originating in the Mucous Membrane of the Jaw:

One, a colored waiter, aged 39, who suffered from chronic mania for eighteen years.

The other, an artist, aged 45, a native of the United States. $\mathrm{He}$ was a demented patient who had had epilepsy for two and a half years.

Both were untidy in their habits and the latter had a history of specific disease. The growth originated three weeks prior to death in the first case, and eight months in the latter. Both died of sapraemia and exhaustion.

Two Patients Died from Epithelioma of the Base of the Tongue and Pharynx:

One, a German, aged 48 , of unknown occupation, an advanced case of dementia, with no definite information as to the previous form of insanity, or of the duration of the growth.

The other, a German merchant, aged 49, of intemperate habits, 
a case of dementia following melancholia. In this case the growth appeared $81 / 2$ months before death, and the insanity four months later, the former being the assigned cause of the latter.

Both died from sapraemia.

One Patient Died of Carcinoma of the Left Orbit:

A Canadian draper, aged 52. He had been operated upon $61 / 2$ weeks before admission, his insanity dating from that time. He was a case of acute mania merging into dementia. Death occurred on the day after admission. The duration of the growth could not be determined.

One Patient Died of Epithelioma of the Side of the Tongue:

A Swiss, aged 52, a demented paretic with a history of specific disease which was the assigned cause of his insanity. Paresis had existed I $1 / 2$ years before the development of the growth, and the patient died four months after its first appearance.

Two Patients Died of Epithelioma of the Face:

One, an Irish tailor, aged 53, of intemperate habits, a case of senile dementia of over five years' duration; the other, a French merchant, aged 68, of intemperate habits, suffering from dementia secondary to melancholia of three years' duration. In both cases the growth developed six weeks prior to death; in both it was very painful and bled easily, and both showed evidence of arterial degeneration of the brain. The immediate cause of death in both cases was exhaustion. The former patient suffered during the last three years of his life from convulsions of an epileptiform character.

Two Patients Died of Epithelioma of the Penis:

One, an Italian waiter, aged 62, a paretic of over three years' duration. The growth developed one month prior to death. The other, an Irish laborer, aged 50, who had undergone privations and been in the almshouse. He was suffering from senile dementia of unknown duration. The growth developed four months prior to death, and was said to have followed chancroid. $\mathrm{He}$ showed evidences of arterio-fibrosis with kidney involvement.

The first patient died of sapraemia, the second of uraemia.

Three Patients Died of Carcinoma of the Rectum:

One, an Irish laborer, aged 75, of temperate habits, a case of 30 
senile dementia of three years' duration. He was hemiplegic and had suffered from epilepsy for twenty-five years. The growth developed one month prior to death.

The second, an Italian laborer, aged 35, of temperate habits, a case of acute mania, passing into dementia, although he had recovered from a previous attack three years before. The carcinoma, following fistula in ano, developed one month prior to death,

The third case occurred in an Irishman, aged 40, a demented imbecile of no occupation. The growth developed six weeks prior to death.

These three cases were similar in the rapid development of the growth, and in the excitement, restlessness and irritability which attended the insanity. All three died of sapraemia.

Two Patients Died of Primary Carcinoma of the Liver:

The first, a laborer, aged 30, a native of the United States, with a history of arterial disease in his mother. He was a case of simple melancholia of unknown duration, who had begun to show symptoms of dementia. Upon admission, he presented evidence of enlargement of the liver, followed by vomiting, ascites and jaundice. He died $21 / 2$ months later of exhaustion.

The other, a French Hebrew, aged 47, an hotel messenger, who had been insane for twenty years, worry being assigned as the cause. He suffered from dementia, secondary to acute mania. The entire surface of the body, except the face and scalp, was covered with eczema, from which he had suffered for years. Two months prior to death he developed symptoms of carcinoma of the liver, jaundice, vomiting, loss of appetite, and diarrhoea. The immediate cause of death was sapraemia.

Two Patients Died of Epithelioma of the Lower Lip:

One, an Irish carpenter, aged 54, who suffered from dementia, secondary to melancholia, of over five years' duration, for which intemperance was the cause assigned. The growth began to develop four years prior to death, the immediate cause of which was exhaustion.

The other case was an English peddler, aged 42, of temperate habits, whose parents both died of asthma. He was a case of acute mania, progressing rapidly into dementia. In this case the 
growth and insanity developed simultaneously, the former being the assigned cause of the latter. The immediate cause of death, which occurred four weeks after the first appearance of the growth, was sapraemia.

Four Patients Died of Primary Carcinoma of the Stomack:

One, a colored patient, aged 20, a native of the United States, of no occupation. He was a case of acute melancholia of unknown duration, which had passed into dementia. Three weeks prior to death, the immediate cause of which was exhaustion, he presented symptoms of carcinoma of the stomach. The growth infiltrated the liver and pancreas.

The second was a German baker, aged 60 , of very intemperate habits, who suffered from senile dementia, the exact duration of which (covering a period of years) was indefinite. He presented symptoms of valvular heart disease and general arterio-fibrosis. The duration of the growth could not be determined. He died of gastric hemorrhage.

The third patient was a German driver, aged 6I, of slightly intemperate habits, who suffered from pulmonary tuberculosis. The insanity and symptoms of the growth appeared simultaneously five weeks prior to death, the immediate cause of which was exhaustion.

The fourth patient was an Irish groom, aged 42, a moderate drinker, who suffered from chronic melancholia of two years' duration. Three months previous to death, the immediate cause of which was exhaustion, he presented symptoms of gastric cancer.

These four cases were similar in the rapid development of the growth; all were cases of agitated melancholia, merging rapidly into dementia, and the insanity in all was characterized by gloominess and depression, suicidal tendencies, delusions of persecution, and vivid hallucinations.

One Patient Died of Sarcoma of the Left Breast:

An Irish cooper, aged 68, of intemperate habits. He suffered from dementia, secondary to melancholia, of $201 / 2$ years' duration; the melancholia followed a left-sided hamiplegia. The tumor was said to have existed many years, but its duration could not be definitely determined. One month prior to death, it began to ulcerate causing intense pain, and he died of exhaustion. 
One Patient Died of Sarcoma of the Right Shoulder:

An Irish salesman, aged 47, who suffered from dementia, secondary to chronic mania and epilepsy of $7^{1} / 2$ years' duration, and who had suffered from an attack of insanity elght years previously. The causes assigned were syphilis, domestic worry and masturbation. Three years prior to death, a sarcoma of the shoulder developed, involving the entire deltoid muscle. A very painful ulceration began in the neoplasm, and the patient died of sapraemia three weeks later.

One Patient Died of Sarcoma of the Liver:

A native of the United States, aged 24, an electrician of intemperate habits, who suffered from dementia secondary to acute mania. His father died of apoplexy. Four months prior to death, he became jaundiced, suffered from pain in the region of the gallbladder, the liver enlarged, ascites supervened, the temperature rose, vomiting and occasional nose-bleed followed. He died of general oedema and exhaustion.

One Patient Died of Osteosarcoma of the Left Knee:

An Irish laborer, aged 44, who had been an inebriate. He was a demented epileptic of the maniacal type. His insanity had existed for more than nine years. The sarcoma existed for more than nine years also, but the exact duration could not be determined. During the last two months of his life, his physical condition failed and the neoplasm enlarged. He died of exhaustion. Five Patients Died of Primary Sarcoma of the Brain:

Three cases were instances of single and two of multiple sarcomata. All were demonstrated by autopsy. The first, in a man aged 40, originated in the dura mater over the sella turcica, and was a small round-celled sarcoma. The second in a man aged $5 \mathrm{I}$, originated in the cerebral falx adjacent to the parietal lobe, and was about 38 millimeters in diameter. The third, in a man aged 34, was a multiple fibrosarcoma of the dura. The larger tumor, 38 millimeters in diameter, was situated near the left Sylvian fissure; the smaller, 12 millimeters in diameter, was situated over the superior occipital convolution near the vertex. The fourth was also a multiple growth of the round spindle-celled variety in a colored patient aged 30 . The larger tumor, 40 millimeters in diameter, originated in the falx cerebri adjacent to the first fron- 
tal convolution, the smaller one from the same structure and was situated immediately alongside of the larger. The fifth occurred in a man aged 57, and was a small round-celled sarcoma, 80 millimeters in diameter, originating in the falciform process of the dura over the crista galli.

The insanity in these five cases was of short duration, varying from $31 / 2$ months to $21 / 4$ years, and was apparently due directly to the presence of the neoplasm. The nature of the insanity in all the cases was much the same and was characterized generally by marked depression, suicidal tendencies, delusions of suspicion, and stuporous states passing into dementia.

In addition to the focal symptoms, which varied with the location of the growth, the principal general symptoms observed were epileptiform convulsions coming on in adult life, a dazed feeling in the head, more pronounced at times then at others, with disturbances of equilibrium, progressive weakness of the lower limbs with a tendency for the legs to give way, the patient falling down helpless, general progressive emaciation of the body, and a wild staring expression of the eyes, with slight exophthalmos and occasional nose-bleed-the last mentioned group being due to the intracranial pressure.

The association of cancer and tuberculosis has been a subject of considerable inquiry. In some cases the one or other preceded and instances have been cited where the two processes existed at the same time in the same glandular structure and it was difficult or impossible to determine the primary disease. Cancerous persons have been believed by some to be the survivors of tubercular families. Of the cases cited three of cancer and one of sarcoma were associated with pulmonary tuberculosis. In all the cancer cases tuberculosis existed prior to the development of the cancer. In one case, the father also suffered from phthisis. In the sarcoma case the history was too indefinite to accurately determine the precedence; the indications, however, pointed to the primary existence of the neoplasm.

In three cases cancer developed in the course of paresis. One patient had a definite specific history, but in all paresis antedated from one and one-half to three years the development of the neoplasm.

Three cases of cancer and one of sarcoma presented definite 
histories of specific disease, although it was highly probable that this number represented only a small proportion of those actually affected, particularly with reference to the cancer cases. The growth in all, however, appeared several years after the primary infection.

Epilepsy existed in two cases of cancer, and in two cases of sarcoma. In all the cases it preceded the development of the neoplasm by from two to twenty-five years.

The association of epilepsy and specific disease existed in one case of cancer and one case of sarcoma. In both, these affections had existed for several years before the development of the neoplasm.

The average age at death of the cancer cases was 48 years, of the sarcoma cases 43 8-9 years. This would seem to indicate that death from cancer occurs at an earlier age in the insane as compared with the population at large. The civil condition presented a marked contrast ; 67.7 per cent of the cancerous cases were single men, whereas 60 per cent of the sarcoma cases were married. Intemperance in the use of alcohol and tobacco was particularly noted in the cases observed, but was a much more prominent feature in those dying from cancer.

The question of heredity has always been considered an important one. This, however, did not assume prominence in the cases observed. In not a single instance was a history of death from cancer or sarcoma in the ancestry obtainable.

It is worthy of note that in those dying of cancer the blonde and brunette types were equally distributed, whereas in the sarcoma cases (with one exception, who was a colored patient), all were of sanguine temperament with light complexions and blue eyes, and mostly of the plethoric type. With one or two exceptions they were strong, active able-bodied men, and particularly so in all the cases of sarcoma of the brain.

The diseases from which they had previously suffered were those incident to plethora-epilepsy, apoplexy, cardiac disease, articular rheumatism. The same class of diseases was traceable in the ancestry and presented somewhat of a contrast to those encountered in the cancer cases, which were principally phthisis, asthma, syphilis, cirrhosis of the liver and kidneys, arteriofibrosis and paresis. 
Impressed by the fact that mesoblastic and epiblastic growths appear to bear some relation to the nutritive and secretory activities of the organism-the former occurring, as a rule, in middleaged, active, able-bodied individuals of sanguine temperament at the beginning of a decline in the nutritive functions; the latter occurring later in life when senile changes have begun, and the vigor of the epithelial structures is passing-and especially impressed by the fact that malignant growths run a more rapid course and occur with greater frequency in the insane, who lack inhibitory control, and in the debilitated; and that cancer occurs more frequently in those whose glandular structures are approaching their functional decline, it occurred to me to inquire whether the tissue changes resulting in an overgrowth were not fundamentally conservators of function to compensate for the waning vigor. The cellular-tissue elements are stintulated by toxic juices rich in substances generated by retrograde metabolic changes, and undergo hyperplasia. The energy of this overgrowth is errant by reason of the failing inhibitory control, and, in some instances, is perhaps whipped into activity by local irritations, congestions or inflammations. A development ad libitum results and uses up all the remaining nutritive powers of the organism to sustain a wanton effort.

If, therefore, the insane suffering from malignant growths, and who present to such a pre-eminent degree those well-recognized evidences of lost inhibitory control associated with grave metabolic tissue changes, were systematically studied, could we not hope to solve the mystery which thus far has cast its shadow upon the achievements of modern medicine? 


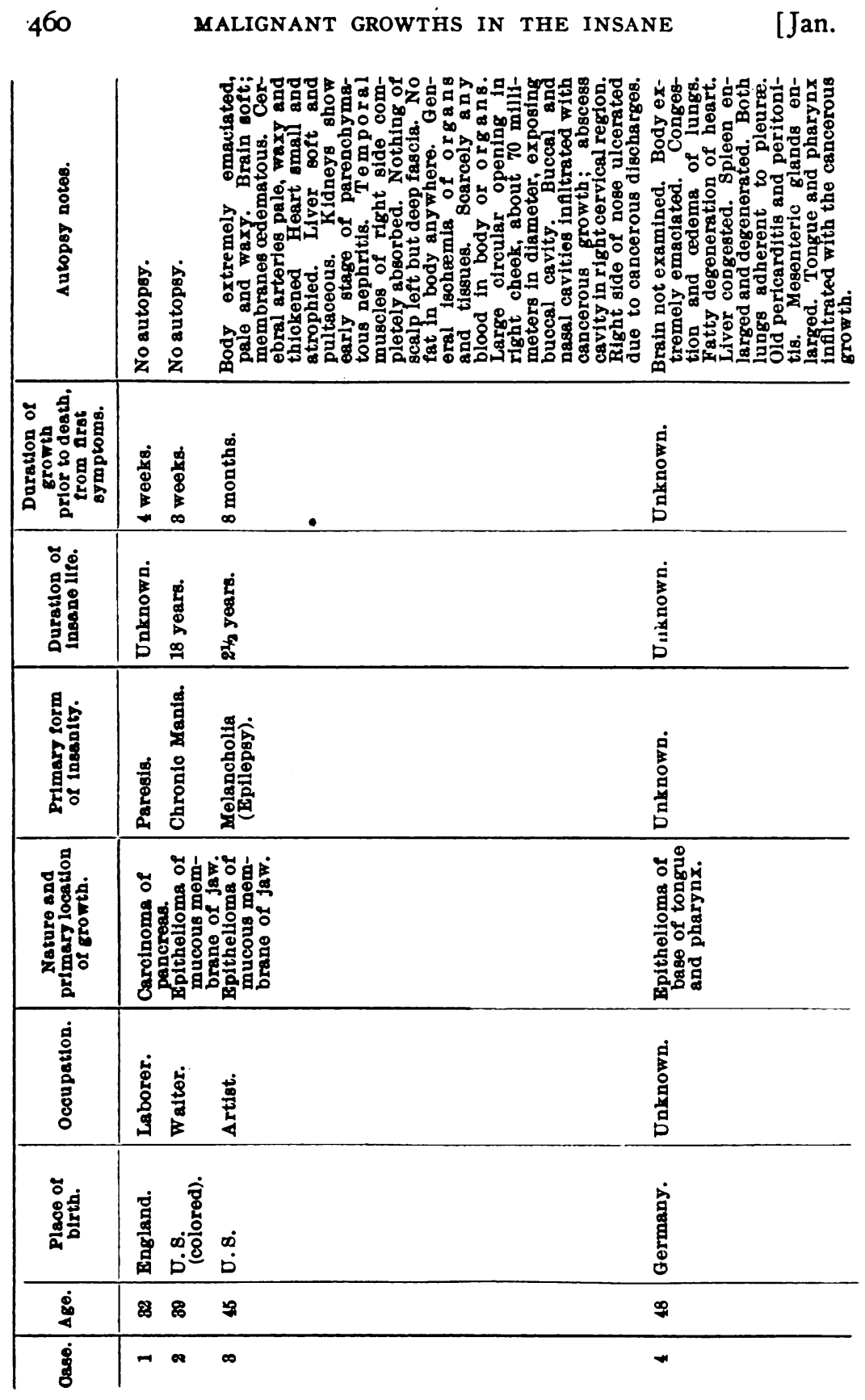




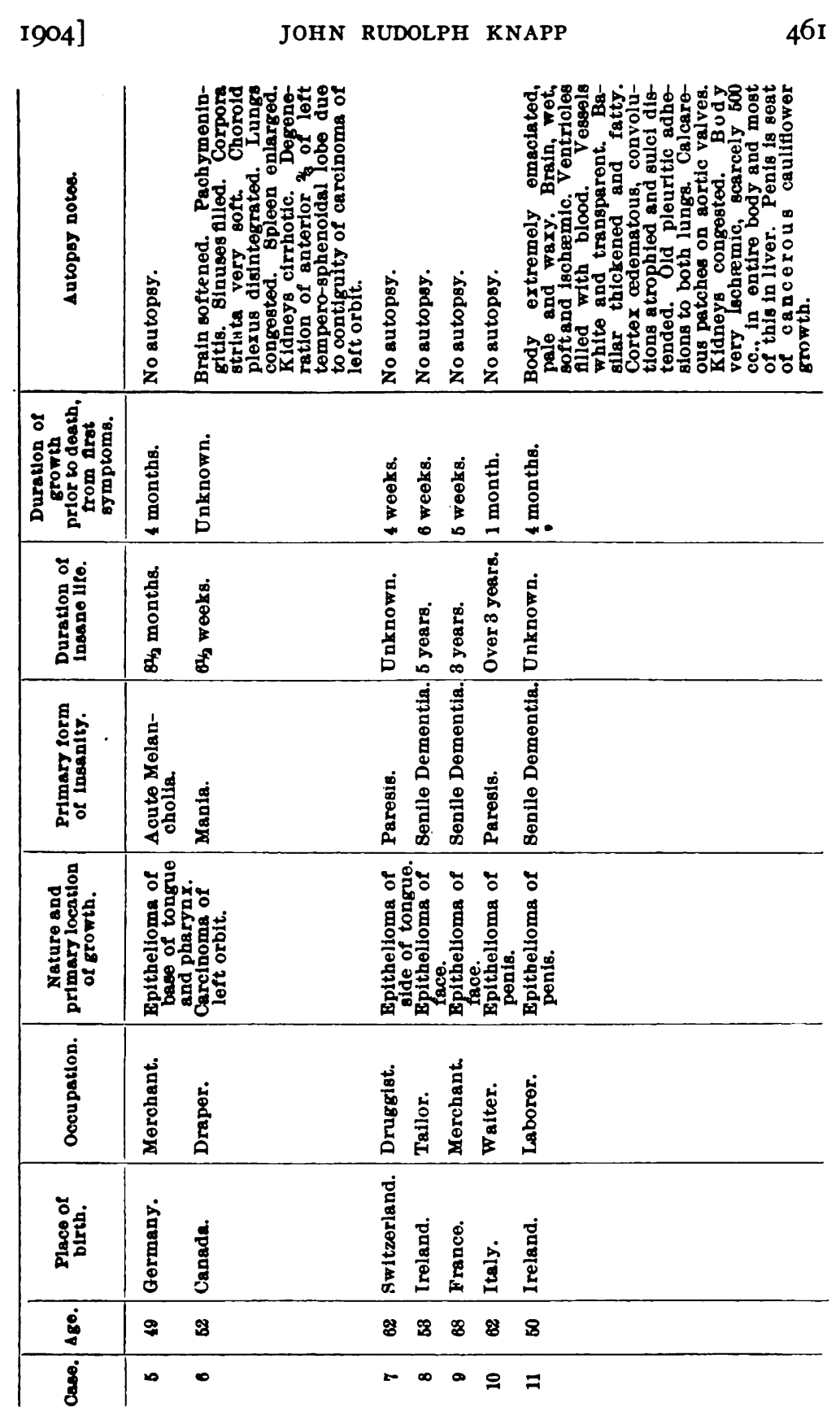




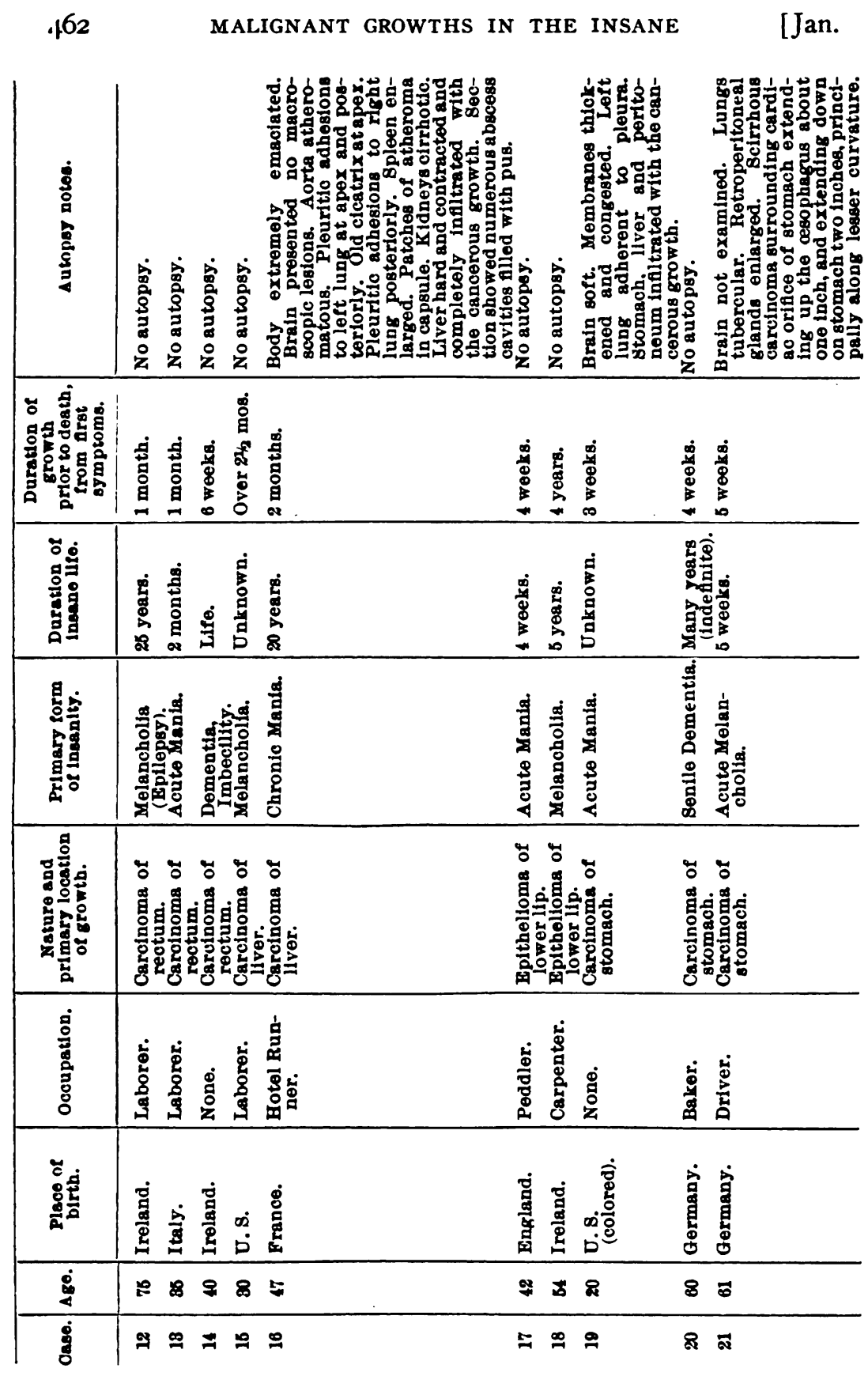




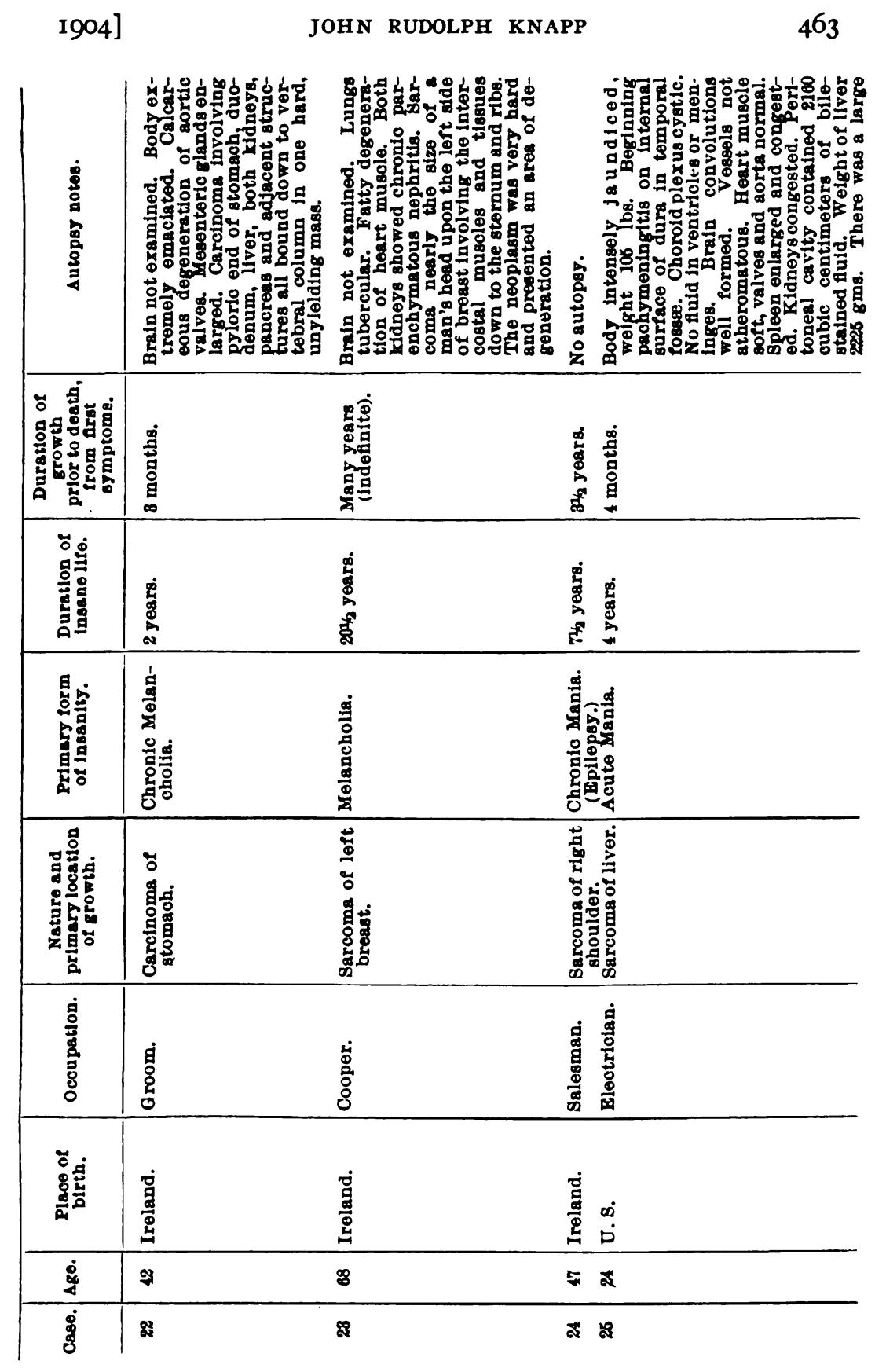




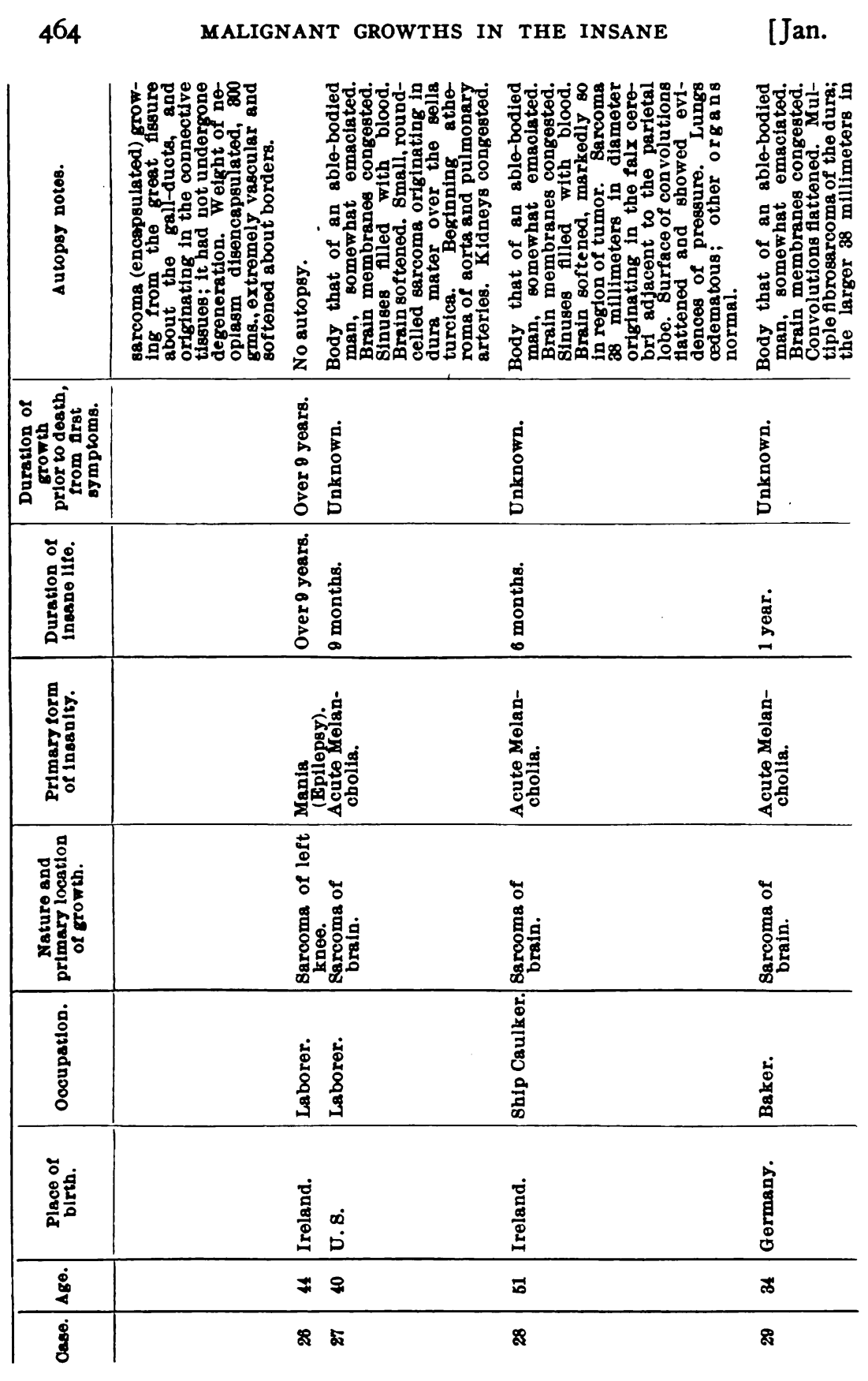




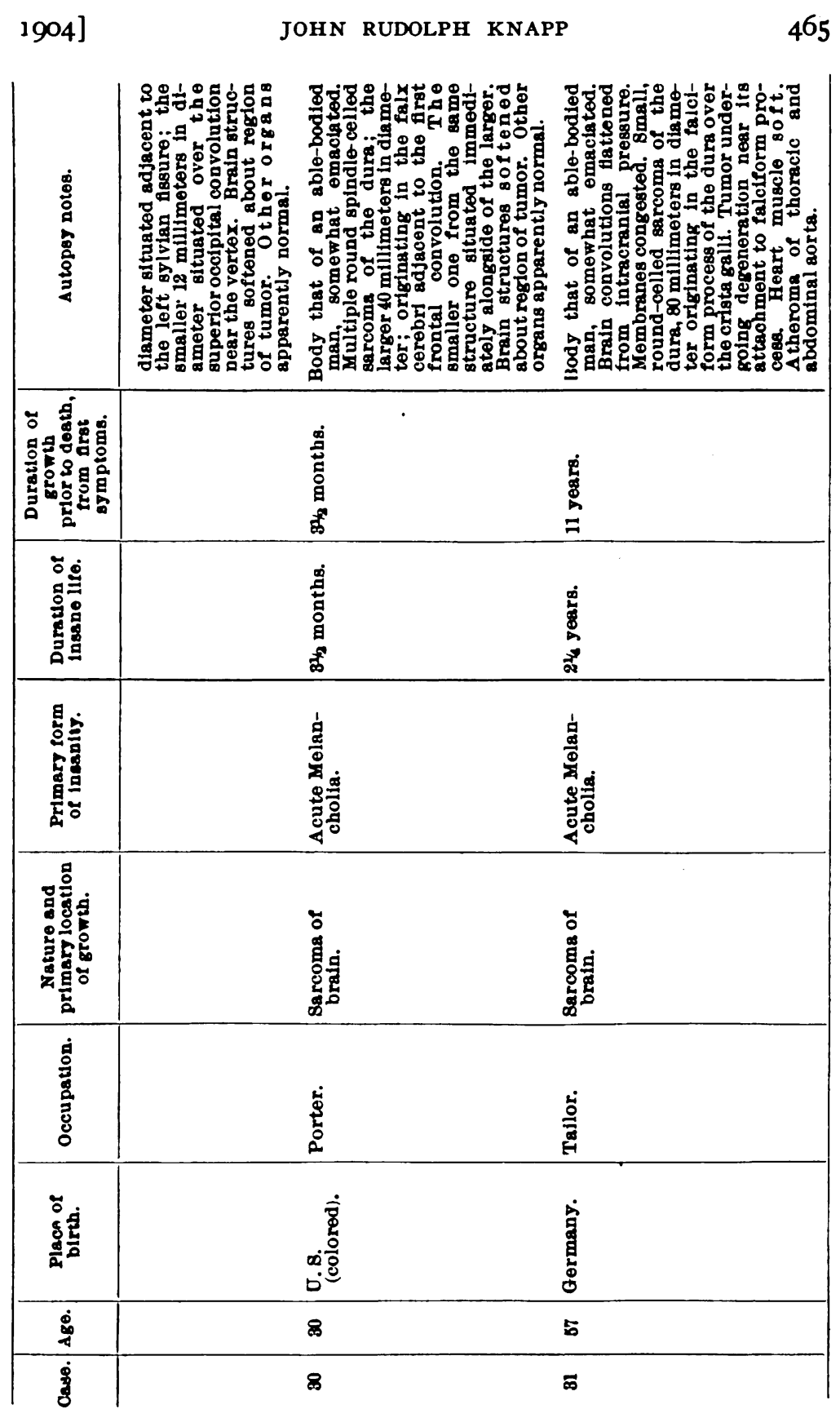




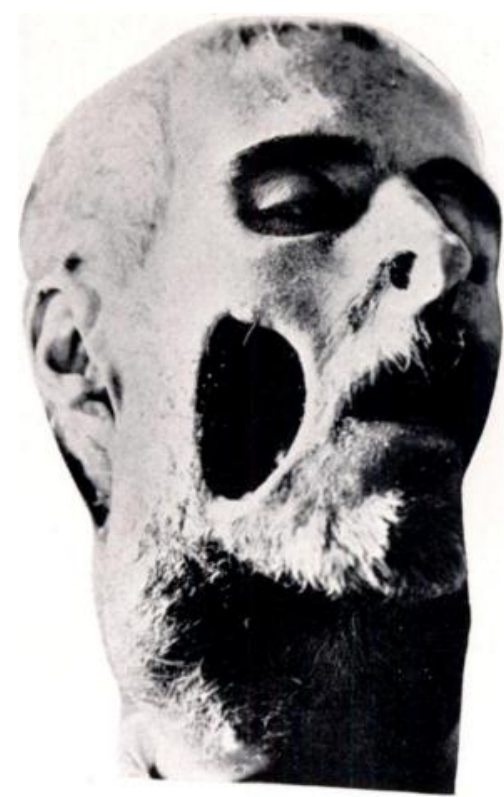

Showing extensive ulceration of the cheek and nose and enlargement of the cervical lymphatic glands in a case of epithelioma of the mucous membrane of the jaw, occurring in a demented epileptic, with definite syphilitic history.

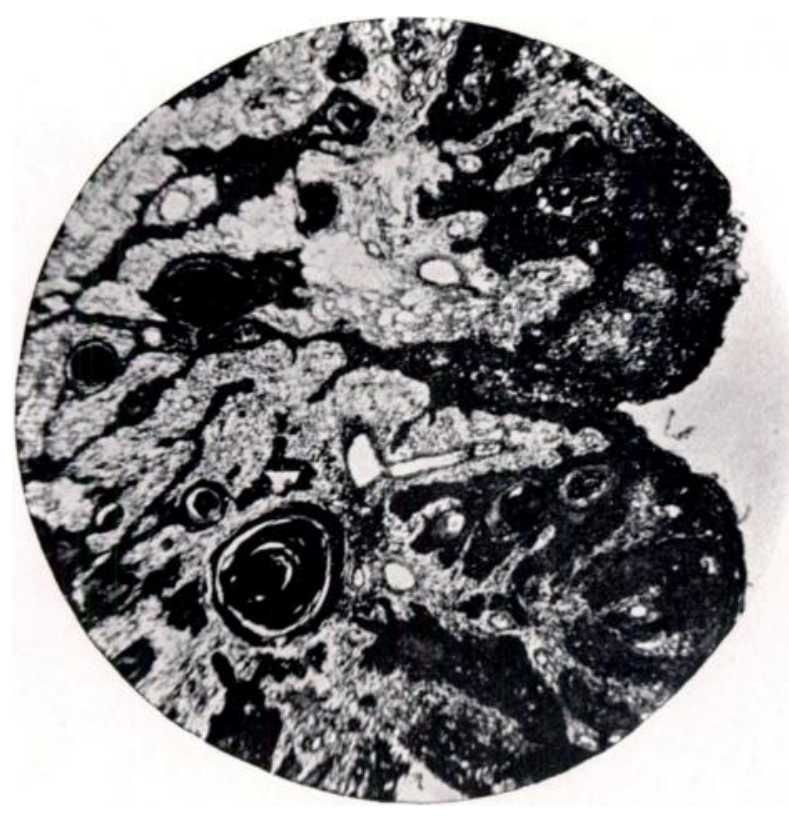

Epithelioma of the mucous membrane of the jaw.

TO ILLUSTRATE DR. JOHN RUDOLPH KNAPP'S PAPER. 


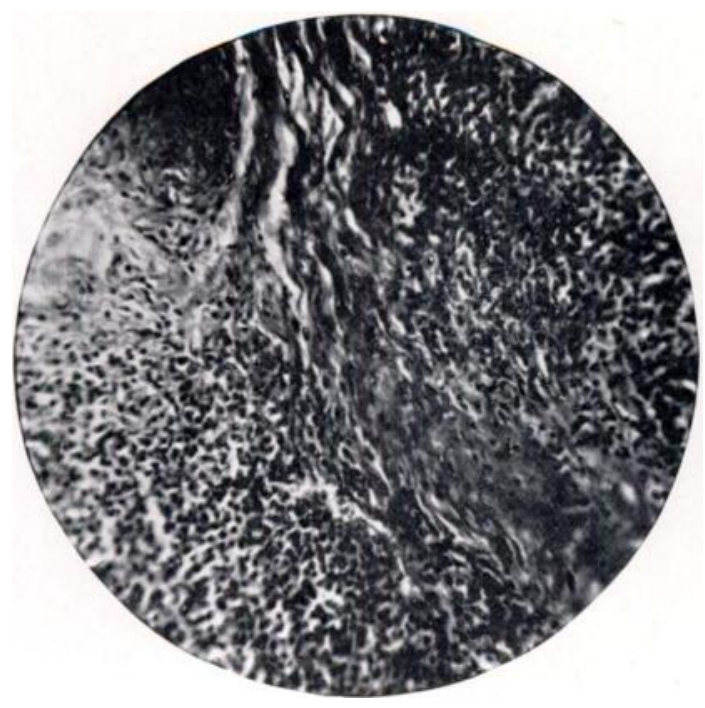

simall Round celled saromat.

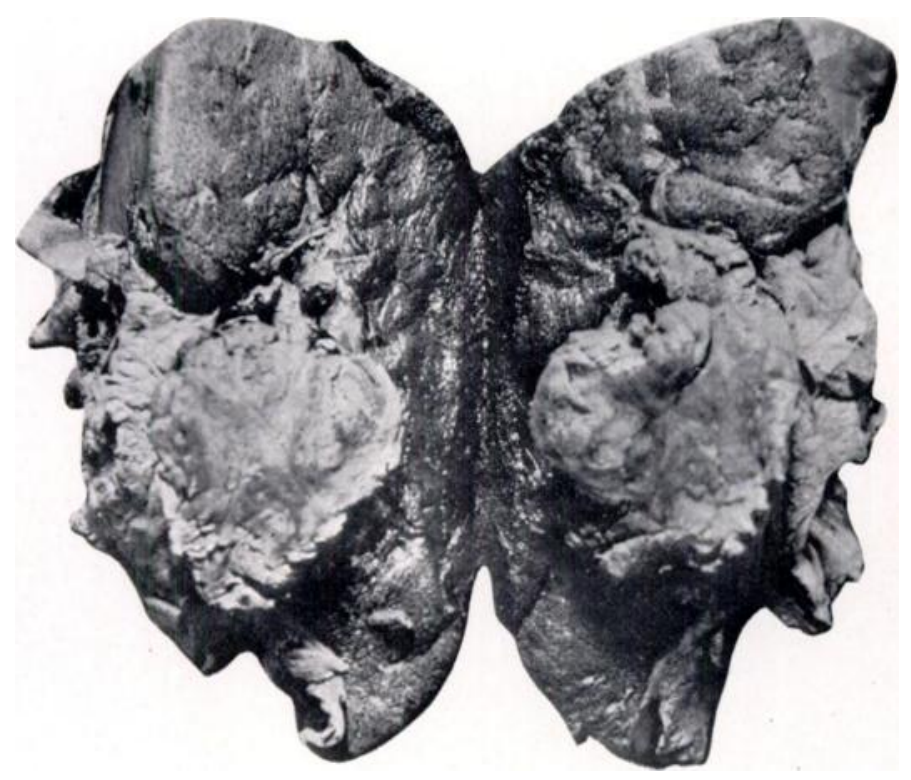

Sarcoma of the Liver.

TO ILLUSTRATE DR. JOHN RUDOLPH KNAPP'S PAPER. 
THE AMERICAN JOURNAL OF INSANITY, VoI. LX, No. 3.

PLATE XV.

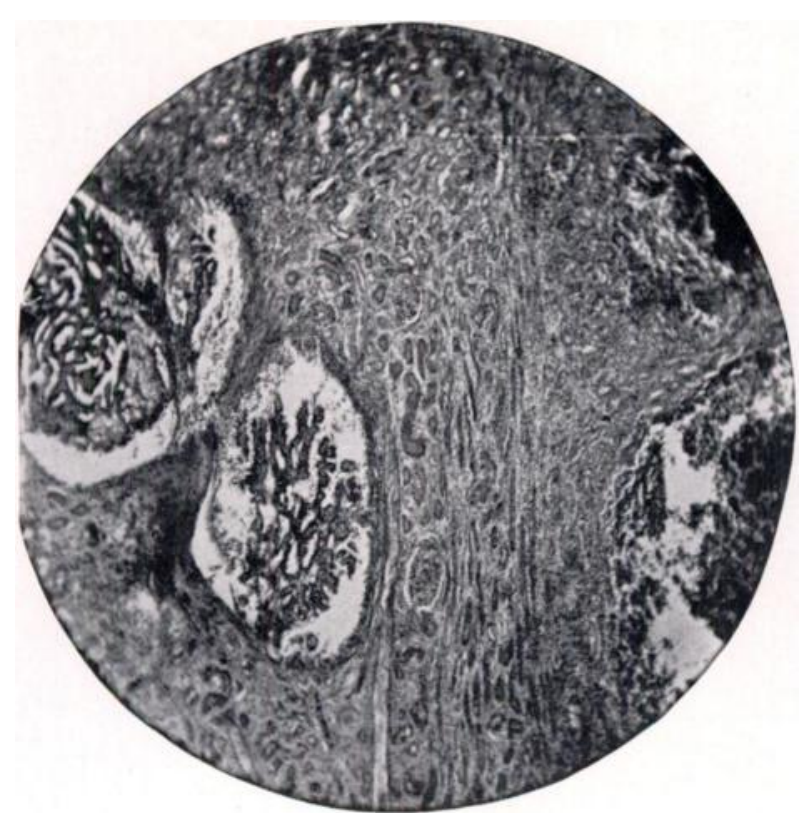

Carcinoma of the Kidney.

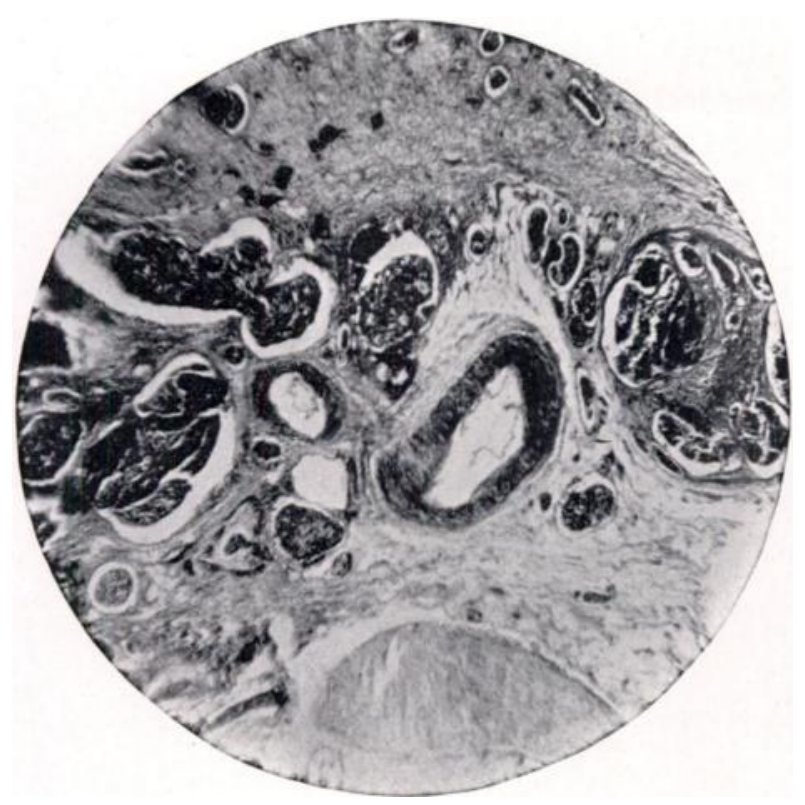

Carcinoma of the Stomach.

TO ILLUSTRATE DR. JOHN RUDOLPH KNAPP'S PAPER. 